DOI: $10.3901 / J M E .2021 .15 .071$

\title{
紧固件防松性能定量评价方法*
}

\author{
张铁亮 $^{1}$ 王 卓 ${ }^{2,3}$ 宋镝冲 $^{2,3}$ 王 磊 $^{2,3}$ 丁晓宇 $^{1}$ \\ (1. 北京理工大学机械与车辆学院 北京 $100081 ;$ \\ 2. 内蒙古第一机械集团有限公司 包头 014030 ;
}

3. 特种车辆及其传动系统智能制造国家重点实验室 包头 014030)

\begin{abstract}
摘要: 工程领域一直都缺少对紧固件防松性能进行定量评价的方法, 针对该问题, 基于现有的横向振动试验方法, 改进了横 向振动试验设备, 提出以导致旋转松动的临界横向力作为评价防松性能的定量指标, 并给出了具体的试验方法和数据获取方 法。基于所提的方法, 对弹簧垫圈、楔形螺母和偏心双螺母的防松性能进行了定量测试与对比, 在本研究试验条件下, 得出 以下结论: (1) 弹簧垫圈没有防松性能; (2) 相同预紧力下, 相比普通螺纹连接, 使用楔形螺母后防松性能最多提升了 $117 \%$; (3) 相同预紧力下, 相比普通螺纹连接, 使用偏心双螺母后防松性能最多提升了 $125 \%$; (4) 在高预紧力条件下, 使用楔形螺 母和偏心双螺母后防松性能和普通螺纹连接的防松性能相差不大。
\end{abstract}

关键词：紧固件; 防松性能; 临界横向力; 横向振动

中图分类号: TG156

\section{Quantitative Evaluation Method of Anti-loosening Performance for Fasteners}

\author{
ZHANG Tieliang $^{1} \quad$ WANG Zhuo $^{2,3}$ SONG Dichong ${ }^{2,3}$ WANG Lei $^{2,3}$ DING Xiaoyu ${ }^{1}$
}

(1. School of Mechanical Engineering, Beijing Institute of Technology, Beijing 100081;

2. Inner Mongolia First Machinery Group Co., Ltd., Baotou 014030;

3. State Key Laboratory of Smart Manufacturing for Special Vehicles and Transmission System, Baotou 014030)

\begin{abstract}
There is a lack of quantitative evaluation method for fastener's anti-loosening performance in engineering field. To solve the problem, based on the existing transverse vibration test method, the transverse vibration test equipment is improved and the critical transverse force that causes rotational loosening is proposed as a quantitative index for evaluating the anti-loosening performance, and detailed test methods and data acquisition methods are given. Based on the method, the anti-loosening performance of spring washer, wedge nut and eccentric double nut are compared. Under the experimental conditions, The results show that: (1) Spring washer has no anti-loosening performance; (2)Under the same preload, compared with common thread connection, the anti-loosening performance of wedge-shaped nut is improved by $116.7 \%$ at most; (3) Under the same preload, compared with the common thread connection, the anti-loose performance of the double eccentric nut is improved by $125 \%$ at most; (4)Under the condition of high preload, the anti-loosening performance of the wedge nut and the eccentric double nut have little difference with the anti-loosening performance of common thread connection.
\end{abstract}

Key words: fasteners; anti-loosening performance; critical transverse force; transverse vibration

0 前言

螺纹连接通常具有结构简单、装拆方便、连接

* 国家自然科学基金(51975055), 国防基础科研核科学挑战专题(TZ2018007) 和天地科技股份有限公司创新创业基金重点(2020-2-TD-ZD002)资助项目。 20200814 收到初稿, 20201212 收到修改稿
可靠、互换性好、价格便宜等优点，在各种机械产 品和工程结构中应用十分广泛。大量的工程经验表 明, 在振动环境下, 螺纹连接容易出现松动失效。 螺纹连接松动失效问题严重影响着机械产品的可靠 性和寿命, 工程中由于螺纹连接松动失效所引发的 质量问题和事故屡见不鲜。为了防止螺纹连接的松 动失效, 人们通常会使用具有防松作用的紧固件, 
如各种防松垫圈和防松螺母。然而, 各种防松紧固 件到底能在多大程度上提升螺纹连接的防松性能 呢? 对于这个基本的问题, 目前并没有准确的答案。 这造成了许多争议, 也给紧固件的设计、制造、选 型及使用带来了许多问题。

目前工程人员通常基于国家标准 $\mathrm{GB} / \mathrm{T}$ $10431-2008^{[1]}$ 或国军标 GJB 715.3A-2002 $2^{[2]}$ 规定的两 种振动试验方法来测试紧固件的防松性能。基于第 一种试验方法, 人们通常根据特定振动周期内 (通常 选用 1500 次)剩余预紧力的多少来评价紧固件的防 松性能; 基于第二种试验方法, 人们可以根据紧固 件在松脱前的持续时间来评价其防松性能。虽然这 两种方法都能在一定程度上对紧固件的防松性能进 行评价, 然而由于试验设备的局限性、数据处理方 法的局限性等多种原因, 它们只能用于定性的对比 不同紧固件防松性能的相对强弱, 或者判断某种紧 固件是否可以通过特定振动条件的考核, 而无法对 紧固件的防松性能进行定量的评价。

对紧固件防松性能进行定量评价的首要难点是 要建立防松性能的定量评价指标(或指标体系), 即 要明确紧固件的防松性能该以何种量纲来评价。本 文采用了国家标准 GB/T 10431-2008 ${ }^{[1]}$ 所推荐的横 向振动试验方法的基本思路, 但改进和完善了试验 设备, 并通过分析预紧力的衰减规律和机理, 提出 了螺纹连接防松性能的定量评价指标和数据获取方 法。基于所提出的方法本文对弹簧垫圈、楔形螺母 (典型产品有 Spiralock®螺母)和偏心双螺母(典型产 品有 HARD-LOCK®螺母)的防松性能进行了定量 测试与对比, 三种紧固件如图 1 所示。其中楔形螺 母和偏心双螺母普遍被认为具有优异的防松性能, 近些年在国内应用越来越广泛。这里需要说明的是, 本文中所述的紧固件仅仅是指用于螺纹连接的紧固 件, 包括各种螺母、螺栓和垫圈, 不包括铆钉、销 子等其他类型的紧固件。

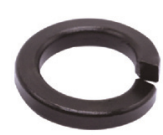

(a) 弹簧垫圈

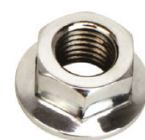

(b) 楔形螺母

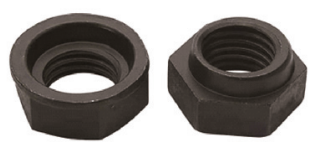

(c) 偏心双螺母
图 1 本研究所用的三种紧固件

\section{1 现有横向振动试验设备局限及改进}

现有国家标准 GB/T 10431-2008 ${ }^{[1]}$ 所采用的横 向振动试验方法最早出现在德国工程师 $\mathrm{JUNKER}^{[3]}$
于 1969 年发表的论文中, JUNKER ${ }^{[3]}$ 通过该试验方 法系统地验证了横向振动载荷容易导致螺纹连接的 松动, 因此该试验方法又常被称为 Junker 测试方法。 Junker 测试方法后来逐渐受到了工业界的广泛认 可, 以它为基础形成了紧固件防松性能测试的标准 方法, 除了我国标准 GB/T 10431-2008 ${ }^{[1]}$, 德国标准 DIN $65151^{[4]}$ 和国际标准 ISO 16130-2015 $5^{[5]}$ 也都是基 于 Junker 测试方法。

图 2 是某个符合国家标准 GB/T 10431-2008 ${ }^{[1]}$ 要求的横向振动试验设备的结构简图。不同厂商所 产该种试验设备的具体结构可能和图 2 不完全相 同, 但是它们的原理都是一致的。图 2 所示的试验 设备主要包括偏心轮、活动板、固定板、预紧力传 感器、横向力测量装置和横向位移传感器。在试验 时, 螺栓和螺母(也可以加垫圈)首先被拧紧到设定 的预紧力, 然后偏心轮在电机作用下产生往复横向 运动, 最大横向位移(即振幅)通过偏心轮的偏心量 控制, 活动板和固定板中间通过埋设滚子等方式尽 量减小摩擦力, 活动板左右往复运动时会给螺纹连 接施加横向力载荷, 横向力可由横向力测量装置 实时记录。在横向振动载荷的作用下, 螺纹连接 会逐渐松动, 预紧力会逐渐下降, 预紧力传感器 可以实时记录振动过程中的预紧力的变化, 试验 时螺纹连接的松动最终会体现为螺母的转动, 而 螺栓端部则被固定板限制无法转动。另外, 在试 验过程中活动板的实际振幅可由横向位移传感器 实时记录。

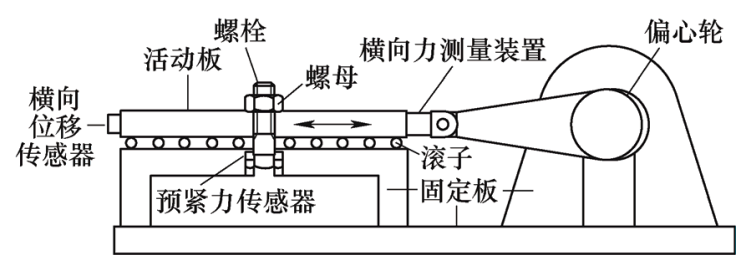

图 2 横向振动试验设备结构简图

图 2 所示的横向振动试验设备只能对振幅进 行开环控制。具体来说, 振幅是通过偏心轮上的 偏心量控制的, 由于连杆、横向力测量装置等结 构在横向力的作用下会压缩或伸长, 这导致螺纹 连接部位所受到的实际振幅和设定偏心量之间的 差距往往很大, 而且是不稳定的。图 3 是某开环 控制的横向振动试验设备在某次测试中所得到的 实际振幅随振动周期的变化情况, 试验样件规格 为 $\mathrm{M} 10$, 初始预紧力为 $25 \mathrm{kN}$, 振动频率为 $12.5 \mathrm{~Hz}$, 设定偏心量为 $1 \mathrm{~mm}$, 而从该图中可以 看出刚开始的实际振幅只有约 $0.4 \mathrm{~mm}$, 且随着振 
动的持续而逐渐增加。

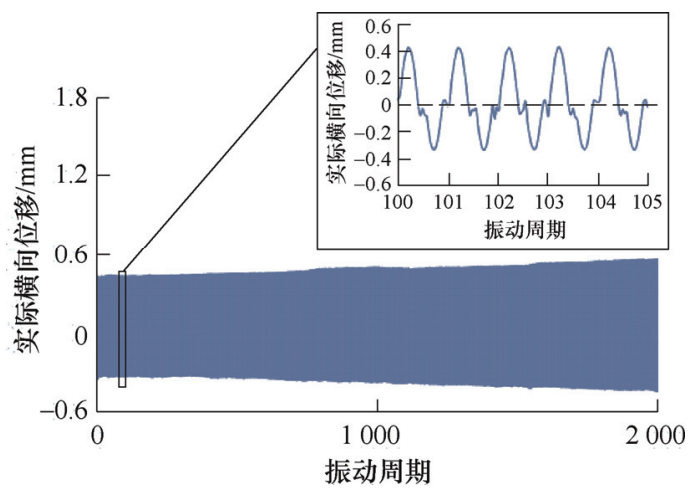

图 3 开环控制的横向振动试验所得到的往复横向位移

螺纹连接所承受的实际振幅和其所承受的横 向力大小紧密相关, 是影响螺纹连接松动的关键 因素。螺纹连接的松动过程是外部因素和内部因 素共同作用的结果, 此时振幅是主要的外部因素, 而紧固件自身的防松能力则是内部因素。既然开 环控制条件下外部因素是不确定的, 那么就无法 从试验结果(即预紧力的衰减数据)中区分出内部 因素的定量影响, 即无法基于试验结果对紧固件 的防松性能进行定量评价。为了解决这一问题, 本文提出了在横向振动试验中对横向力进行闭环 控制的理念, 并改进了现有的横向振动试验设备, 使之具有了横向力闭环控制的功能。改进后的试 验设备中横向力测量装置的最大量程为 $50 \mathrm{kN}$, 预紧力测量装置的最大量程为 $100 \mathrm{kN}$, 横向位移 测量装置的最大量程为 $10 \mathrm{~mm}$, 上述三个量的示 值误差均不超过标定值的 $0.5 \%$ 。

图 4 为改进的横向振动试验设备结构简图, 该试验设备是基于横向力实测数据调整液压伺服 作动器的控制参数, 从而实现对横向力的闭环控 制; 另外, 该设备也可以基于横向位移的实测数 据来实现对横向位移的闭环控制。图 5 是改进的 横向振动试验设备在某次测试中所得到的实际横 向力随振动周期的变化情况, 试验样件规格 M10, 初始预紧力为 $18 \mathrm{kN}$, 振动频率为 $5 \mathrm{~Hz}$, 设定横 向力是 $1 \mathrm{kN}$, 从实测结果可以看出该设备对横向 力有较好的控制精度。图 6 是改进的横向振动试 验设备在某次测试中所得到的实际横向位移随振 动周期的变化情况, 试验样件规格 M10, 初始预 紧力为 $25 \mathrm{kN}$, 振动频率为 $5 \mathrm{~Hz}$, 设定振幅是 $0.5 \mathrm{~mm}$, 从实测结果可以看出该设备对横向位移 也有较高的控制精度。本文后续试验都是基于改进 的横向振动试验设备完成。

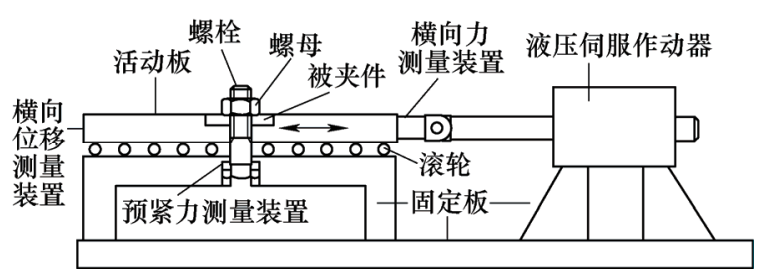

图 4 改进的横向振动试验设备结构简图

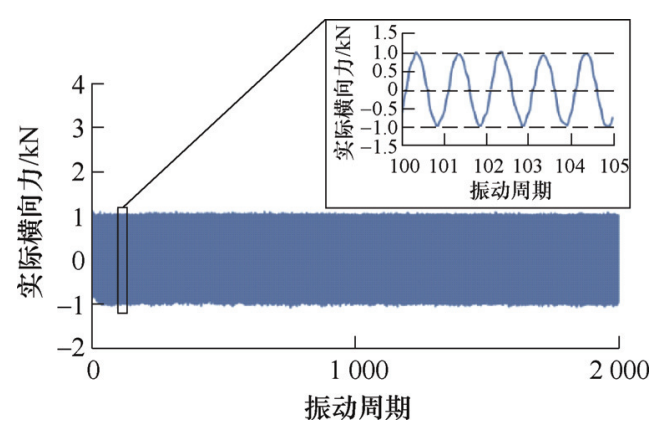

图 5 改进的设备对横向力的控制效果

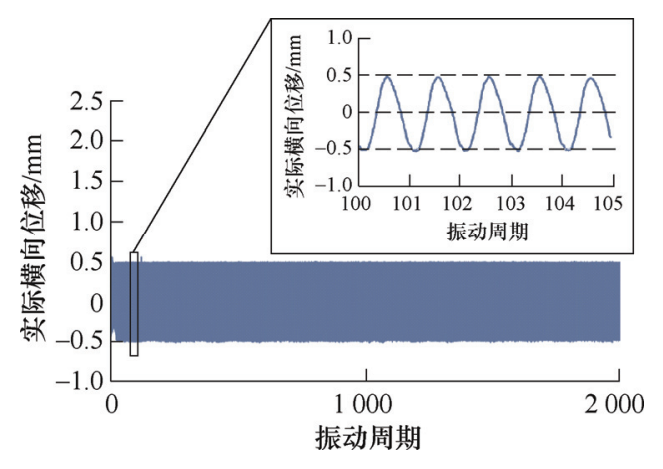

图 6 改进的试验设备对横向位移的控制效果

\section{2 螺纹连接防松性能定量评价指标 和数据获取方法}

为了对紧固件防松性能进行定量评价, 需要建 立科学的螺纹连接防松性能定量评价指标。由于预 紧力的衰减是多种因素综合作用的结果, 要准确评 判防松性能需要把不同因素对预紧力衰减的影响区 分开。已有研究已经证明 ${ }^{[6-11]}$ : 在横向振动试验条 件下, 螺纹连接典型的预紧力衰减过程可以分为三 个阶段, 如图 7 所示。在第一阶段, 预紧力会发生 非线性的快速衰减，这一阶段导致预紧力衰减的主 要原因是材料的塑性变形, 同时也有应力再分布、 旋转松动和微动磨损等因素的影响。在第二阶段中, 预紧力近似呈现线性衰减, 这一阶段导致预紧力衰 减的主要原因为内外螺纹间的旋转松动, 而其他因 素影响相对较小。在第三阶段, 螺纹连接结构会逐 渐出现疲劳裂纹, 导致预紧力衰减速度加快, 甚至 
突然断裂。图 8 为基于改进试验设备的某实测的预 紧力衰减曲线, 试验所用样件规格为 M10, 初始预 紧力为 $25 \mathrm{kN}$, 振幅 $0.5 \mathrm{~mm}$, 频率 $12.5 \mathrm{~Hz}$, 可以从 该图中清晰看出上述的三个阶段。

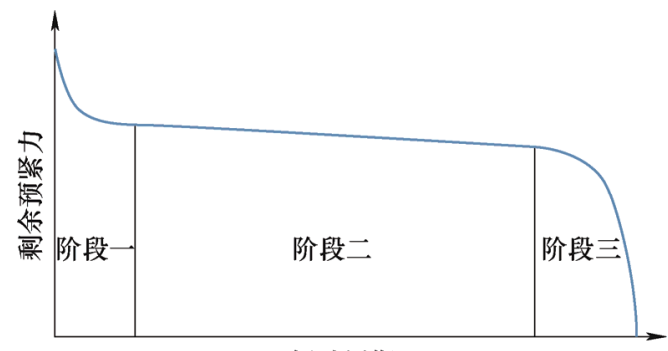

图 7 典型预紧力衰减曲线

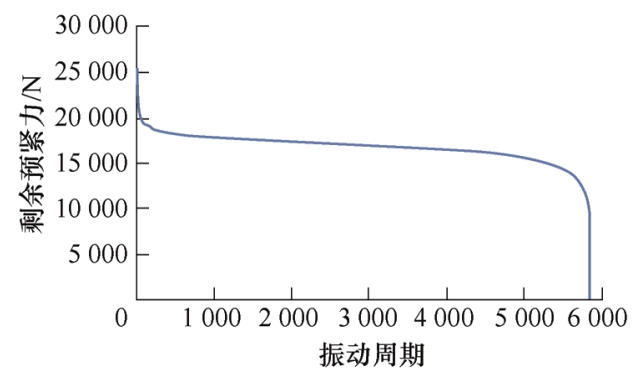

图 8 某实测预紧力衰减曲线

为了从图 7 所示的预紧力衰减数据中获得对紧 固件防松性能的定量评价结果, 我们需要明确 “松 动” 和 “防松性能” 的准确含义。螺纹连接的松动 分为旋转松动和非旋转松动, 所谓旋转松动是指内 外螺纹间发生了导致预紧力衰减的刚性转动, 它对 应着图 7 中的第二阶段; 所谓非旋转松动是指除旋 转松动之外其他因素导致的预紧力衰减, 图 7 中第 一阶段和第三阶段主要受非旋转松动的影响。我们 把 “防松性能” 定义为防止螺纹连接产生旋转松动 的能力, 它和预紧力衰减过程第二阶段的数据密切 相关。之所以这么定义防松性能是因为防松紧固件 的作用主要是防止旋转松动, 而难以抑制非旋转松 动导致的预紧力衰减。

基于上述定义, 在理想情况下, 当图 7 所示曲 线的第二阶段的斜率为零时, 意味着螺纹连接没有 发生旋转松动; 当第二阶段斜率小于零时, 意味着 发生了旋转松动, 且斜率的绝对值越大, 旋转松动 就越剧烈。而且已有研究 ${ }^{[7]} 已$ 经证明: 横向力存在 一个临界值, 当螺纹连接所受横向力小于该临界值 时, 螺纹连接不发生旋转松动, 当螺纹连接所受横 向力大于该临界值时, 螺纹连接则会发生旋转松动, 因此可以用横向力的临界值(或称临界横向力)作为 防松性能的定量评价指标, 对紧固件(或螺纹连接)
的防松性能进行定量评价。需要说明的是, 该指标 是基于本文所述的横向振动试验条件提出的, 不适 用于其他形式的外载荷。

图 9 是某次试验所得的不同横向力下的预紧力 衰减曲线, 样件规格为 M10, 初始预紧力为 $18 \mathrm{kN}$, 频率为 $5 \mathrm{~Hz}$ 。从图 9 可以看出: 当横向力较小时, 如 $1.2 \mathrm{kN}$ 时, 预紧力在第二阶段的变化曲线近乎水 平, 此时没有观测到螺纹连接的旋转松动; 而当横 向力为 $1.3 \mathrm{kN}$ 时, 斜率绝对值显著增加, 螺纹连接 出现了明显的旋转松动。

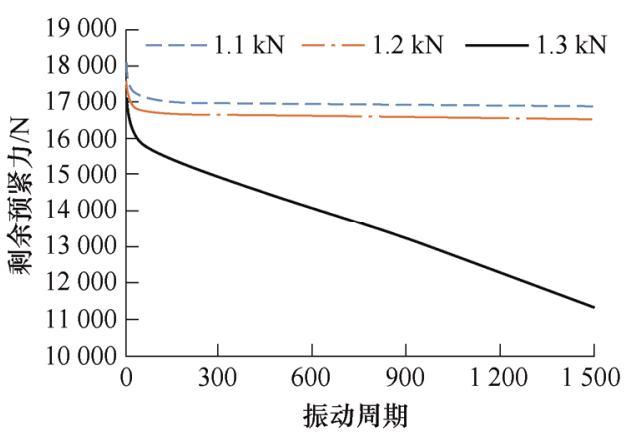

图 9 不同横向力下的预紧力衰减曲线

图 9 所示的试验结果说明临界横向力的确是存 在的, 但是想要准确确定临界横向力的数值, 仍然 有两个问题需要解决: (1)由于预紧力是逐渐变化的, 我们无法准确确定不同阶段间的转折点, 因此基于 哪个周期区间的数据来获取第二阶段斜率值(或其 绝对值)是一个需要解决的问题; (2)理想情况下, 当 第二阶段的斜率为零时, 意味着螺纹连接没有发生 旋转松动, 而实际上由于磨损、温度变化、测量误 差、数据处理误差等因素的影响, 实测的第二阶段 斜率不会完全为零, 因此如何基于实测斜率准确寻 找旋转松动发生的临界点也是一个需要解决的问 题。下面将对这两个问题的解决方法进行具体说明。

为了定量获取第二阶段斜率绝对值, 本文提出 一种方法: 如图 10 所示, 首先, 每隔特定周期 $n$, 将所测预紧力随振动周期变化曲线分成 $m$ 段; 然后 基于最小二乘法，对任意第 $i$ 段曲线进行线性拟合， 从而得到第 $i$ 段曲线的斜率绝对值 $a_{i}$, 如公式 1 所 示, 式中 $j$ 表示第 $j$ 个振动周期, $\overline{j_{i}}$ 为第 $i$ 段曲线 中振动周期的平均值, $y_{j}$ 为第 $j$ 个振动周期时的剩余 预紧力, $\overline{y_{i}}$ 为第 $i$ 段曲线中剩余预紧力的平均值。

$$
a_{i}=\left|\frac{\sum_{j=1+(i-1) n}^{i n} j y_{j}-n \overline{j_{i}} \bar{y}_{i}}{\sum_{j=1+(i-1) n}^{i n} j^{2}-n \bar{j}_{i}^{2}}\right| \quad i=1,2, \cdots, m
$$




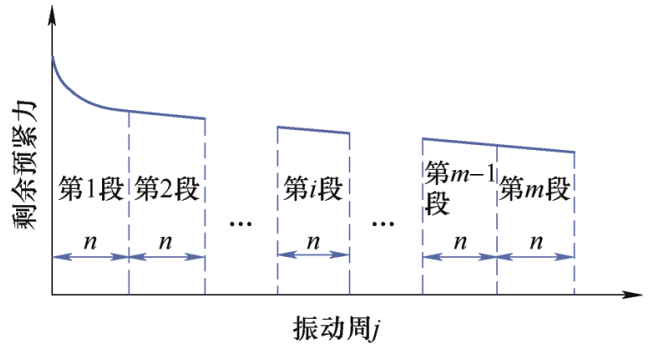

图 10 预紧力衰减曲线分段方法

图 11 是基于图 9 中横向力为 $1.1 \mathrm{kN}$ 时的预 紧力变化数据进行拟合所得到的一系列斜率绝对 值, 周期间隔 $n$ 为 300 。在所有的斜率绝对值中, 可以选取最小的值作为预紧力衰减曲线第二阶段 斜率绝对值, 单位为 $\mathrm{N} /$ 周期, 即对应图 11 中的 $A$ 点。为了对比不同预紧力下的结果, 可以对斜率 绝对值进行归一化处理, 即将所得斜率绝对值除 以该组试验初始预紧力的大小, 并乘以 $100 \%$, 得 到归一化斜率, 单位为\%/周期, 该处理过程可以 用公式 2 表达, 式中 $k$ 为最终求得的归一化斜率, $F_{0}$ 为初始预紧力。

$$
k=\left(\min \left(a_{i}, i=1,2, \cdots, m\right) / F_{0}\right) \%
$$

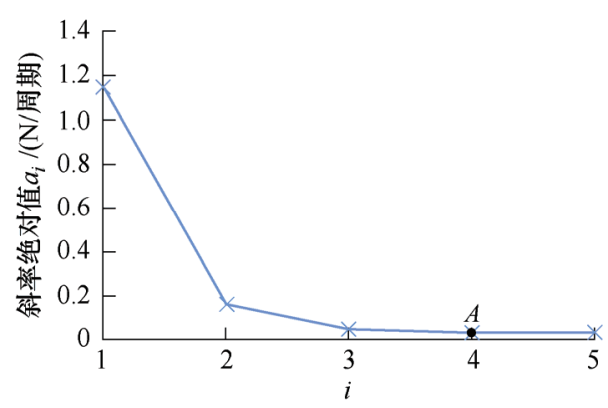

图 11 基于预紧力衰减曲线拟合得到 的斜率绝对值

得到了归一化斜率之后, 需要根据归一化斜 率来找到旋转松动发生的临界点, 从而确定临界 横向力。此时, 需要选取一个合理的斜率阈值, 当实际归一化斜率大于该阈值时, 就认为发生了 旋转松动。

为了选取合理的阈值, 需要进行一定的试验摸 索。本文基于大量的试验研究, 总结归纳出典型的 横向力与归一化斜率的关系曲线如图 12 所示。从图 12 可以看出, 该曲线大致可以分为 $A$ 和 $B$ 两个阶段, 在 $A$ 阶段, 归一化斜率值较小, 且随着横向力增加, 归一化斜率值会缓慢增加, 此时并未发生明显的旋 转松动, 预紧力衰减可能主要是磨损等其他因素导
致的; 在 $B$ 阶段, 随着横向力增加, 归一化斜率值 迅速增加, 意味着螺纹连接发生了明显的旋转松动。 因此曲线从 $A$ 阶段到 $B$ 阶段的转折处对应着旋转松 动开始发生的临界状态, 该转折处对应的归一化斜 率值即可作为判断旋转松动是否发生的斜率阈值, 如图 12 中虚线所示。

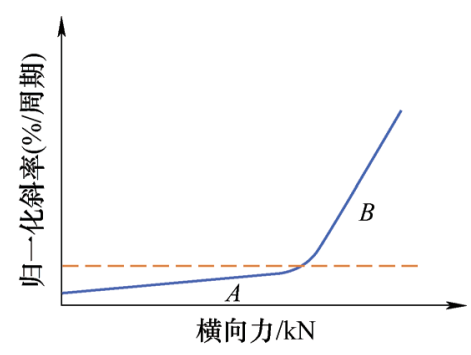

图 12 典型的横向力与归一化斜率关系曲线

\section{3 试验设计}

基于前述的试验方法、防松性能定量评价指标 和数据获取方法, 本文设计并完成了一组试验来定 量测试弹簧垫圈、楔形螺母和偏心双螺母的防松性 能。试验所用样件信息如表 1 所示, 其中普通螺栓 符合 GB/T 5783-2016 ${ }^{[12]}$ 标准, 普通螺母符合 GB/T 6170-2015 $5^{[13]}$ 标准, 在试验开始前对所有试验样件进 行了超声清洗。

表 1 试验样件参数

\begin{tabular}{cccc}
\hline 试验样件 & 规格 & 材料 & 表面处理 \\
\hline 普通螺栓 & 10.9 级 M10 & $20 \mathrm{MnTiB}$ & 发黑 \\
普通螺母 & 10 级 M10 & 45 钢 & 发黑 \\
弹簧垫圈 & 10 级 M10 & $65 \mathrm{Mn}$ & 发黑 \\
楔形螺母 & 10 级 M10 & 碳钢 & 镀锌 \\
偏心双螺母 & 8 级 M10 & $\mathrm{S} 45 \mathrm{C}$ & 磷酸盐皮膜 \\
\hline
\end{tabular}

试验根据所用紧固件种类的不同分为了 4 大 组, 如表 2 所示, 其中大组 1 为对照组, 其余大 组通过与大组 1 进行对比来定量分析防松性能, 对于偏心双螺母而言(大组 4), 由于其由两个螺母 (凸螺母和凹螺母)组成, 因此对拧紧工艺有一定 的要求, 本研究中的偏心双螺母拧紧工艺参考李 天雷等人的研究 ${ }^{[14]}$, 具体过程不再赘述。每大组 试验根据初始预紧力不同又分为了 6 小组, 每小 组试验又包含若干不同的横向力条件, 如图表 3 所示, 表中横向力的采样间隔为 $0.1 \mathrm{kN}$ 。每一种 
试验件下需要做三次重复性试验, 每个试验样品 只使用一次。

表 2 各组试验采用的紧固件种类

\begin{tabular}{cccc}
\hline 大组 1 & 大组 2 & 大组 3 & 大组 4 \\
\hline 普通螺栓+普 & 普通螺栓+普通螺 & 普通螺栓+楔形 & 普通螺栓+偏心 \\
通螺母 & 母+弹簧垫圈 & 螺母 & 双螺母 \\
\hline
\end{tabular}

表 3 试验条件

\begin{tabular}{ccccccc}
\hline & 小组 1 & 小组 2 & 小组 3 & 小组 4 & 小组 5 & 小组 6 \\
\hline 初始预紧力 $/ \mathrm{kN}$ & 14 & 18 & 22 & 26 & 34 & 38 \\
振动周期/次 & 1600 & 1600 & 1600 & 1600 & 1600 & 1600 \\
振动频率 $/ \mathrm{Hz}$ & 5 & 5 & 5 & 5 & 5 & 5 \\
横向力范围 $/ \mathrm{kN}$ & $0.9 \sim 2.5$ & $1.1 \sim 2.8$ & $1.4 \sim 3.4$ & $1.9 \sim 3.9$ & $2.6 \sim 4$ & $3.2 \sim 4.2$ \\
重复试验次数/次 & 3 & 3 & 3 & 3 & 3 & 3 \\
\hline
\end{tabular}

在进行上述试验前, 需要先进行一定的试验 来确定斜率阈值, 然后根据确定的阈值来判断旋 转松动是否发生, 当某一试验条件下有一次或一 次以上的试验所得的归一化斜率大于斜率阈值 时, 则认为该试验条件下已经发生了旋转松动, 只有当三次重复性试验所得的归一化斜率都小 于斜率阈值时, 才认为该试验条件下没有发生旋 转松动。

\section{4 试验结果}

\section{1 斜率阈值的确定}

在试验中发现, 尽管螺纹连接未出现旋转松动, 但第二阶段的归一化斜率也可能会随着预紧力的增 大而增大，此时斜率的增大主要是由磨损等非旋转 松动因素导致的, 预紧力越大, 磨损等因素的影响 也会越大。为了充分考虑磨损等因素的影响, 可以 在较大的预紧力下获得前文所述的斜率阈值。本试 验中采用的最大预紧力为 $38 \mathrm{kN}$, 因此可以基于 38 $\mathrm{kN}$ 下的试验数据确定斜率阈值。基于前文所述的归 一化斜率获取方法对 $38 \mathrm{kN}$ 下的试验数据进行处 理, 可以得到表 2 中 4 种连接结构的归一化斜率值 随横向力变化曲线, 如图 13 所示。基于第 2 节所 述的斜率阈值获取方法, 从图 13 可以看出, 归一 化斜率在大于 $0.01 \% /$ 周期之后出现了较快的增 加, 因此统一选取 $0.01 \% /$ 周期作为旋转松动判断 斜率阈值是合适的, 本文试验最终使用的斜率阈 值为 $0.01 \% /$ 周期。
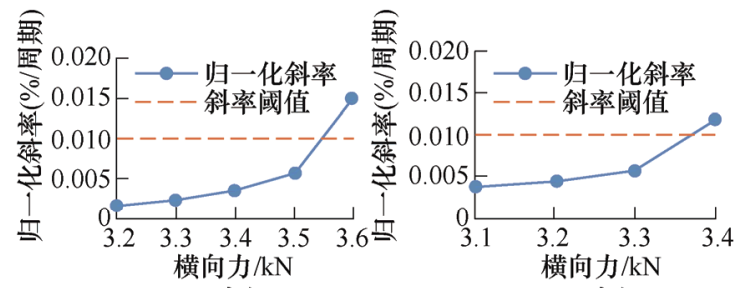

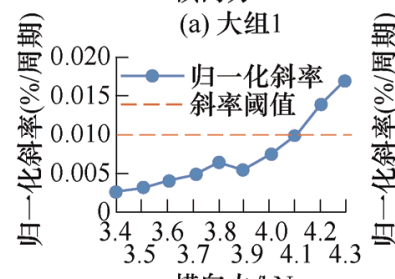

横向力 $/ \mathrm{kN}$

(c) 大组 3

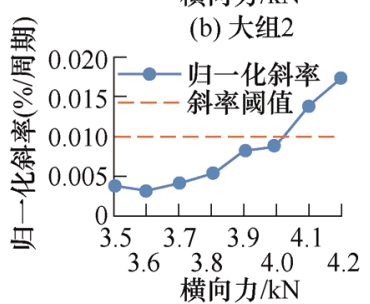

(d) 大组 4
图 13 四种连接结构在不同横向力下的归一化斜率

\section{2 基于临界横向力的防松性能定量评价}

以大组 1 中的小组 1 为例, 所得到的不同横向 力下的归一化斜率如图 14 所示。依据前述的判断标 准, $1.1 \mathrm{kN}$ 时三个样件均没有发生旋转松动, $1.2 \mathrm{kN}$ 时有两个样件发生了旋转松动, 因此可知该种条件 下的临界横向力大约介于 $1.1 \mathrm{kN}$ 和 $1.2 \mathrm{kN}$ 之间, 本 文近似取 $1.1 \mathrm{kN}$ 作为该种条件下的临界横向力。

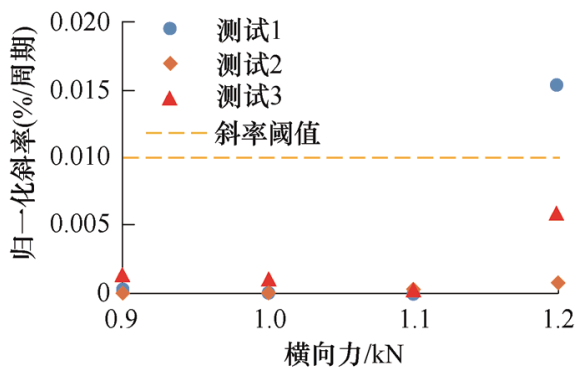

图 14 不同横向力下的归一化斜率

用同样的方法，可以得到 4 种螺纹连接结构在 6 种初始预紧力条件下的临界横向力, 结果如图 15 所示, 从该图可以看出, 随着预紧力增加, 所有螺 纹连接结构的临界横向力均在增加, 说明了增加预 紧力可以有效提升防松性能。除预紧力外, 现有研 究 ${ }^{[7,15-18]}$ 已经证明装夹长度、摩擦因数、材料参数等 因素也会对螺纹连接临界横向力即防松性能产生 影响。

基于图 15 所示结果, 可以定量分析和对比弹簧 垫圈、楔形螺母和偏心双螺母的防松性能。具体地, 把使用三种防松紧固件时的临界横向力(第 2、3、4 大组结果)与普通螺纹连接的临界横向力 (第 1 大组 结果)分别相减, 获得三种防松紧固件所产生的临界 横向力增加值, 即为防松性能的增加值。把临界横 向力的增加值与普通螺纹连接的临界横向力相除, 
可以得到使用三种防松紧固件后的临界横向力的提 升百分比, 即为防松性能提升百分比。

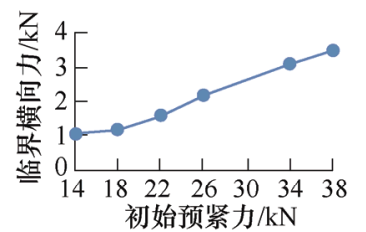

(a) 大组 1

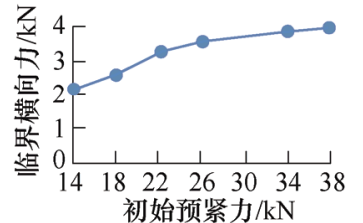

(c) 大组 3

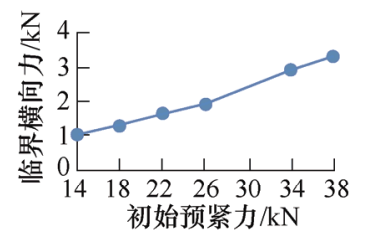

(b) 大组 2

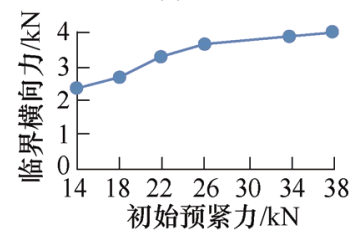

(d) 大组 4
图 154 种螺纹连接在 6 种不同预紧力下的临界横向力

图 16 为使用弹簧垫圈后的防松性能提升百分 比, 可以看到防松性能提升百分比基本在 $0 \%$ 处波 动, 波动范围为 $-13.6 \%$ 到 $8.3 \%$ 。图 16 中的数据波 动很大程度上是由横向力采样间隔产生的测量误 差、不同样件的摩擦因数不一致等因素导致的。因 此尽管图 17 的数据有波动, 但不影响得出结论: 在 本试验条件下, 相比于普通螺纹连接, 使用弹簧垫 圈后防松性能没有出现令人信服的提升效果, 因此 认为弹簧垫圈没有防松效果。

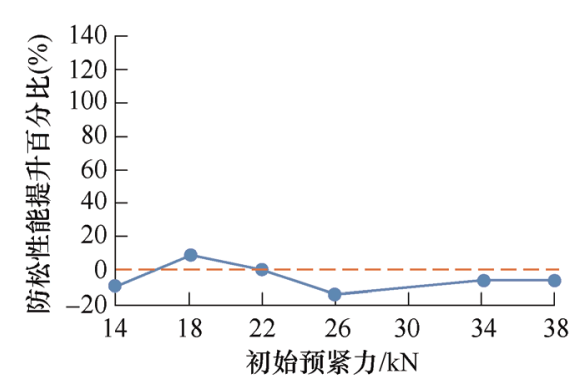

图 16 使用弹簧垫圈后防松性能提升百分比

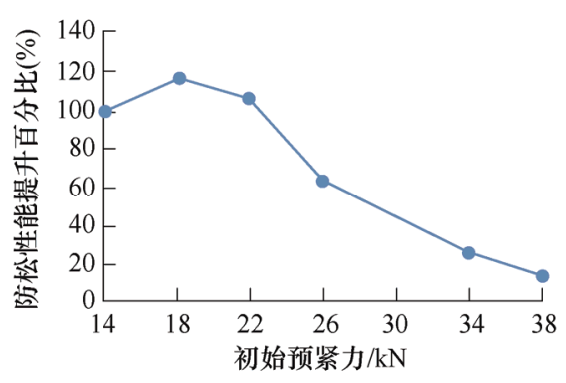

图 17 使用楔形螺母后防松性能提升百分比

图 17 为使用楔形螺母后防松性能提升百分比, 可以看出, 在本研究试验条件下, 使用楔形螺母后, 防松性能最多提升约 $117 \%$ 。此外从该图也可以看 出, 当预紧力较小时, 使用楔形螺母后防松性能提
升显著, 但当预紧力较大时, 使用楔形螺母后防松 性能的提升效果较小, 在预紧力 $38 \mathrm{kN}$ 的情况下, 提升百分比约为 $14 \%$, 说明此时防松性能和普通螺 纹连接相差不大。

图 18 为使用偏心双螺母后防松性能提升百 分比, 从图中可以看出, 在本研究试验条件下, 使用偏心双螺母后，防松性能最多提升约 $125 \%$ 。 此外从该图也可以看出, 当预紧力较小时, 使用 偏心双螺母后防松性能提升显著, 而当预紧力较 大时, 使用偏心双螺母后防松性能的提升效果较 小, 在预紧力 $38 \mathrm{kN}$ 的情况下, 防松性能提升百 分比约为 $14 \%$, 说明此时防松性能和普通螺纹连 接相差不大。

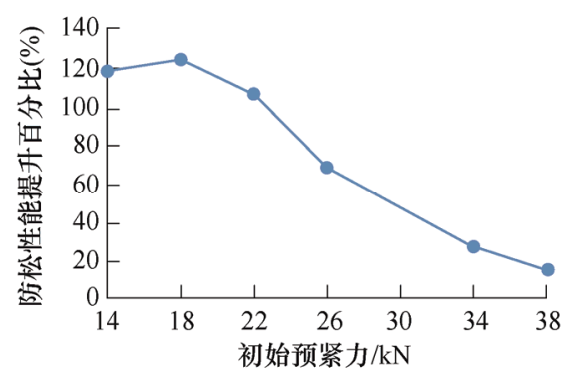

图 18 使用偏心双螺母后防松性能提升百分比

\section{5 结论}

本文改进了现有的横向振动试验设备, 使其具 备横向推力闭环控制的功能, 以此为基础提出以导 致螺纹连接发生旋转松动的临界横向力作为评价防 松性能的定量指标, 并给出了具体的数据获取方法, 基于所提的方法，本文对弹簧垫圈、楔形螺母和偏 心双螺母的防松性能进行了定量测试与对比, 在本 研究试验条件下, 得出以下几点结论。

(1) 在相同预紧力下, 相比普通螺纹连接, 使 用弹簧垫圈后防松性能提升数值基本在 $0 \%$ 处波动, 说明弹簧垫圈没有防松性能。

(2) 在相同预紧力下, 相比于普通螺纹连接, 使用楔形螺母后防松性能最多提升了 $117 \%$;

(3) 在相同预紧力下, 相比于普通螺纹连接, 使用偏心双螺母后防松性能最多提升了 $125 \%$ 。

(4) 在高预紧力条件下, 使用楔形螺母和偏心 双螺母后防松性能和普通螺纹连接的防松性能相差 不大。

需要说明的是, 上述结论的得出是基于本文 的试验条件, 当螺栓规格、涂层、装夹长度等试 验条件不同于本文时, 得出的结论可能不同, 但 
本文所述的紧固件防松性能评价方法是具有普适 性的。此外, 理论上来讲, 横向力和横向位移存 在一定的对应关系, 既然存在临界横向力, 相应 的也应该存在一个临界横向位移(即临界振幅), 因此使用临界横向位移作为防松性能定量评判指 标也具有理论上的可行性, 但具体效果仍需进一 步研究。

\section{参 考 文 献}

[1] 中华人民共和国国家质量监督检验检疫总局, 中国国家 标准化管理委员会. GB/T 10431-2008 紧固件横向振 动试验方法 [S]. 北京: 中国标准出版社, 2009.

General Administration of Quality Supervision, Inspection and Quarantine of the People's Republic of China,

Standardization Administration. GB/T 10431-2008 Transverse vibration testing method for fasteners[S]. Beijing: Standards Press of China, 2009.

[2] 国防科学技术工业委员会. GJB 715.3A-2002 紧固件 试验方法 振动[S]. 北京: 国防科工委军标出版发行部, 2002.

Commission of Science, Technology and Industry for National Defence. GJB 715.3A-2002 Fastener test methods vibration[S]. Beijing : Military Standard Publication Department of Commission of Science Technology and Industry for National Defense, 2002.

[3] JUNKER G. New Criteria for self-loosening of fasteners under vibration[J]. SAE Transactions, 1969, 78(1): 314-335.

[4] Deutsches Institut für Normung e.V.. DIN 65151 Aerospace series-dynamic testing of the locking characteristics of fasteners under transverse loading conditions [S]. Germany: German Engineering Association, 2002.

[5] International Organization for Standardization. ISO 16130-2015 Aerospace series - dynamic testing of the locking behaviour of bolted connections under transverse loading conditions (vibration test)[S]. 2015.

[6] JIANG Y, ZHANG M, LEE C. A study of early stage self-loosening of bolted joints[J]. Journal of Mechanical Design, 2003, 125(3): 518-526.

[7] GONG H, LIU J, DING X. Study on the critical loosening condition toward a new design guideline for bolted joints[J]. Proceedings of the Institution of Mechanical Engineers Part C-Journal of Mechanical Engineering Science, 2019, 233(9): 3302-3316.

[8] 巩浩, 刘检华, 丁晓宇. 振动条件下螺纹预紧力衰减机
理和影响因素研究 [J]. 机械工程学报, 2019, 55(11): 138-148.

GONG Hao, LIU Jianhua, DING Xiaoyu. Study on the mechanism and influencing factors of preload decline for bolted joints under vibration[J]. Journal of Mechanical Engineering, 2019, 55(11): 138-148.

[9] PAI N, HESS D. Experimental study of loosening of threaded fasteners due to dynamic shear loads[J]. Journal of Sound and Vibration， 2002， 253(3): 585-602.

[10] PAI N, HESS D. Three-dimensional finite element analysis of threaded fastener loosening due to dynamic shear load[J]. Engineering Failure Analysis, 2002，9(4): 383-402.

[11] GONG H, LIU J, DING X. Study on the mechanism of preload decrease of bolted joints subjected to transversal vibration loading $[\mathrm{J}]$. Part B : Journal of Engineering Manufacture, 2019, 233(12): 2320-2329.

[12] 中华人民共和国国家质量监督检验检疫总局, 中国国家 标准化管理委员会. GB/T 5783-2016 六角头螺栓 全 螺纹[S]. 北京: 中国标准出版社, 2016.

General Administration of Quality Supervision, Inspection and Quarantine of the People's Republic of China,

Standardization Administration. GB/T 5783-2016 Hexagon head bolts - full thread[S]. Beijing: Standards Press of China, 2016.

[13］中华人民共和国国家质量监督检验检疫总局, 中国国家 标准化管理委员会. GB/T 6170-2015 1 型六角螺母[S]. 北京: 中国标准出版社，2015.

General Administration of Quality Supervision, Inspection and Quarantine of the People's Republic of China,

Standardization Administration. GB/T 6170-2015 Hexagon nuts, style 1[S]. Beijing: Standards Press of China, 2015.

[14] 李天雷, 刘锴, 孙文东, 等. 振动工况下的双螺母结构 防松性能研究 $[J]$. 宇航总体技术，2018，2(4)：52-58.

LI Tianlei, LIU Kai, SUN Wendong, et al. Study on anti-loosening performance of double nut joint under vibration condition[J]. Astronautical Systems Engineering Technology, 2018, 2(4): 52-58.

[15] GONG H, LIU J, DING X. Thorough understanding on the mechanism of vibration-induced loosening of threaded fasteners based on modified Iwan model[J]. Journal of Sound and Vibration, 2020, 473: 115238.

[16] GONG H, LIU J, DING X. Study on local slippage accumulation between thread contact surfaces and novel anti-loosening thread designs under transversal 
vibration[J]. Tribology International, 2021, 153: 106558

[17] 刘检华, 巩浩, 丁晓宇. 振动条件下楔形垫圈的防松性 能研究 [J]. 振动与冲击, 2019, 38(5): 38-44.

LIU J, GONG H, DING X. Anti-loosening performance of wedge washers under vibration[J]. Journal of Vibration and Shock, 2019, 38(5): 38-44.

[18] LIU J, GONG H, DING X. Effect of ramp angle on the anti-loosening ability of wedge self-locking nuts under vibration[J]. Journal of Mechanical Design-Transactions of the ASME，2018，140(7): 072301.

作者简介: 张铁亮, 男, 1996 年出生。主要研究方向为连接力学。 E-mail: bitzhangt1@163.com 丁晓宇(通信作者), 男, 1984 年出生, 博士, 副教授。主要研究方向为 接触力学、连接力学、复杂结构精密装配理论与技术。

E-mail: xiaoyu.ding@bit.edu.cn 